\title{
The effect of deep inspiration breath-hold technique on left anterior descending coronary artery and heart dose in left breast irradiation
}

\author{
Pitchaya Sakyanun ${ }^{1,2}$, Kitwadee Saksornchai $^{1,2}$, Chonnipa Nantavithya ${ }^{1,2}$, Chakkapong Chakkabat ${ }^{1,2}$, \\ Kanjana Shotelersuk ${ }^{1,2}$ \\ ${ }^{1}$ Division of Radiation Oncology, Department of Radiology, Faculty of Medicine, Chulalongkorn University, Bangkok, Thailand \\ ${ }^{2}$ Division of Radiation Oncology, Department of Radiology, King Chulalongkorn Memorial Hospital, Thai Red Cross Society, Bangkok, Thailand
}

Received: February 21, 2020

Revised: July 5, 2020

Accepted: August 7, 2020

Correspondence:

Kanjana Shotelersuk

Division of Radiation Oncology,

Department of Radiology, King

Chulalongkorn Memorial Hospital,

1873 Rama IV Road, Pathumwan,

Bangkok 10330, Thailand.

Tel: +66-2-256-4334

Fax: +66-2-256-459

E-mail: kanjana.s@chula.ac.th

ORCID:

https://orcid.org/0000-0002-8714-5733
Purpose: To determine the effect of the deep inspiration breath-hold (DIBH) technique on left anterior descending coronary artery (LAD) region and heart dose in left breast cancer irradiation.

Materials and Methods: Twenty-five left breast cancer patients who previously received breast-conserving surgery underwent computed tomography (CT) simulation with both free-breathing (FB) and $\mathrm{DIBH}$ techniques and four radiation treatment plans. The plan comprised the following with both the FB and DIBH techniques: whole breast (WB), and WB with internal mammary lymph nodes (WB+IMNs). The prescription dose was 50 Gy in 25 fractions. The doses to the LAD region, heart and lungs were compared. Moreover, in-field maximum heart distance (maxHD) and breast volume were analyzed for correlations with the mean heart dose (MHD).

Results: In the WB plan with DIBH vs. FB techniques, the mean radiation doses to the LAD region, $M H D$, and the left lung $V_{20}$ were 11.48 Gy vs. $19.84 \mathrm{~Gy}(\mathrm{p}<0.0001), 2.95 \mathrm{~Gy}$ vs. $5.38 \mathrm{~Gy}(\mathrm{p}<0.0001)$, and $19.72 \%$ vs. $22.73 \%(p=0.0045)$, respectively. In the WB+IMNs plan, the corresponding values were 23.88 Gy vs. $31.98 \mathrm{~Gy}(\mathrm{p}<0.0001), 6.43 \mathrm{~Gy}$ vs. $10.24 \mathrm{~Gy}$ ( $p<0.0001)$, and $29.31 \%$ vs. $32.1 \%$ ( $p$ $=0.0009)$, respectively. MHD correlated with $\operatorname{maxHD}(r=0.925)$ and breast volume $(r=0.6)$.

Conclusion: The use of the DIBH technique in left breast cancer irradiation effectively reduces the radiation doses to the LAD region, heart and lungs. MHD is associated with maxHD and breast size.

Keywords: Breast cancer, Radiation, Coronary vessels, Heart dose, Deep inspiration breath-hold

\section{Introduction}

Breast cancer is the most common female cancer among women in Thailand and worldwide $[1,2]$. With technical advancements, the treatment outcome, quality of life and survival of breast cancer patients have been much improved. Currently, radiotherapy remains an important modality in breast cancer treatment. Radiation therapy either after breast-conserving surgery or postmastectomy significantly reduces recurrence risk and mortality [3,4]. Recently, internal mammary node (IMN) irradiation has been increasingly used after its overall survival benefit was shown $[5,6]$.

Although breast irradiation improves survival in breast cancer patients, cardiac complications are of concern especially in left breast irradiation. Adding IMN irradiation with a wide tangential field could increase the radiation exposure to the heart. Darby et al. [7] reported that the rate of major coronary events increased by 7.4\% per Gray (Gy) of the mean radiation dose to the heart (mean heart dose [MHD]) without a minimum dose threshold. MHD has been commonly used when evaluating the effect of radiation on the heart. Recently, the radiation dose to left anterior descending coronary artery

Copyright (C) 2020 The Korean Society for Radiation Oncology

This is an Open Access article distributed under the terms of the Creative Commons Attribution Non-Commercial License (http://creativecommons.org/licenses/by-nc/4.0/) which permits unrestricted non-commercial use, distribution, and reproduction in any medium, provided the original work is properly cited. 
(LAD) and other substructures has also been studied [8].

Aiming to minimize radiation doses to normal tissues in breast cancer patients, the deep inspiration breath-hold (DIBH) technique has been extensively studied [9]. The DIBH technique expands the lungs and moves diaphragm downward, which in turn moves the heart posteriorly and inferiorly. This maximizes the distance between the chest wall and the heart during or close to deep inspiration; consequently, reduces radiation exposure to the heart.

Effects of the DIBH technique in reducing the radiation dose to the LAD have recently been reported [10-13]; however, delineation of the LAD requires an injection of a contrast agent, which is not usually performed in our clinical practice. We contoured the anterior interventricular groove from its origin down to the apex of the heart as the LAD region. This study intended to study the effect of the $\mathrm{DIBH}$ technique on reducing the radiation dose to the LAD region and heart in left breast cancer patients.

\section{Materials and Methods}

\section{Patient selection and simulation}

After obtaining approval from the Institutional Review Board of the Faculty of Medicine, Chulalongkorn University (No. 409/60), we prospectively enrolled patients with left sided breast cancer who underwent breast-conserving surgery, had a good performance status (Eastern Cooperative Oncology Group [ECOG] 0-1), and could perform breath holding. Either Philips Brilliance Big Bore or Siemens SOMATOM Definition CT (computed tomography) was used for simulation in our division. In the CT simulation, a breast board was used to immobilize the patients while both arms were up. The patients underwent two series of CT simulation scans; free-breathing (FB) and DIBH without an intravenous contrast injection (Fig. $1 A, 1 B)$. Radiopaque markers were placed on the patient's chest to indicate the field borders. The medial and lateral borders were at the midline and mid-axillary line, respectively. The upper border was the lower border of the clavicular head, and the lower border was approximately 1-2 cm below the inframammary fold. A CT scan during FB was performed first, followed by a DIBH scan. For the DIBH scan, the patients were trained to perform a deep inspiration and then hold their breath for approximately 20 seconds. We provided a teaching video clip to the patients so that they could study the concept of the DIBH and practice how to hold their breath during CT scans and treatments [14]. The stability of patient's breath holding status was monitored by the Varian Real-time Position Management (RPM) respiratory gating system during the CT simulation and by the AlignRT (VisionRT, London, UK) during daily treatments. The CT slice thickness was $3 \mathrm{~mm}$, and the
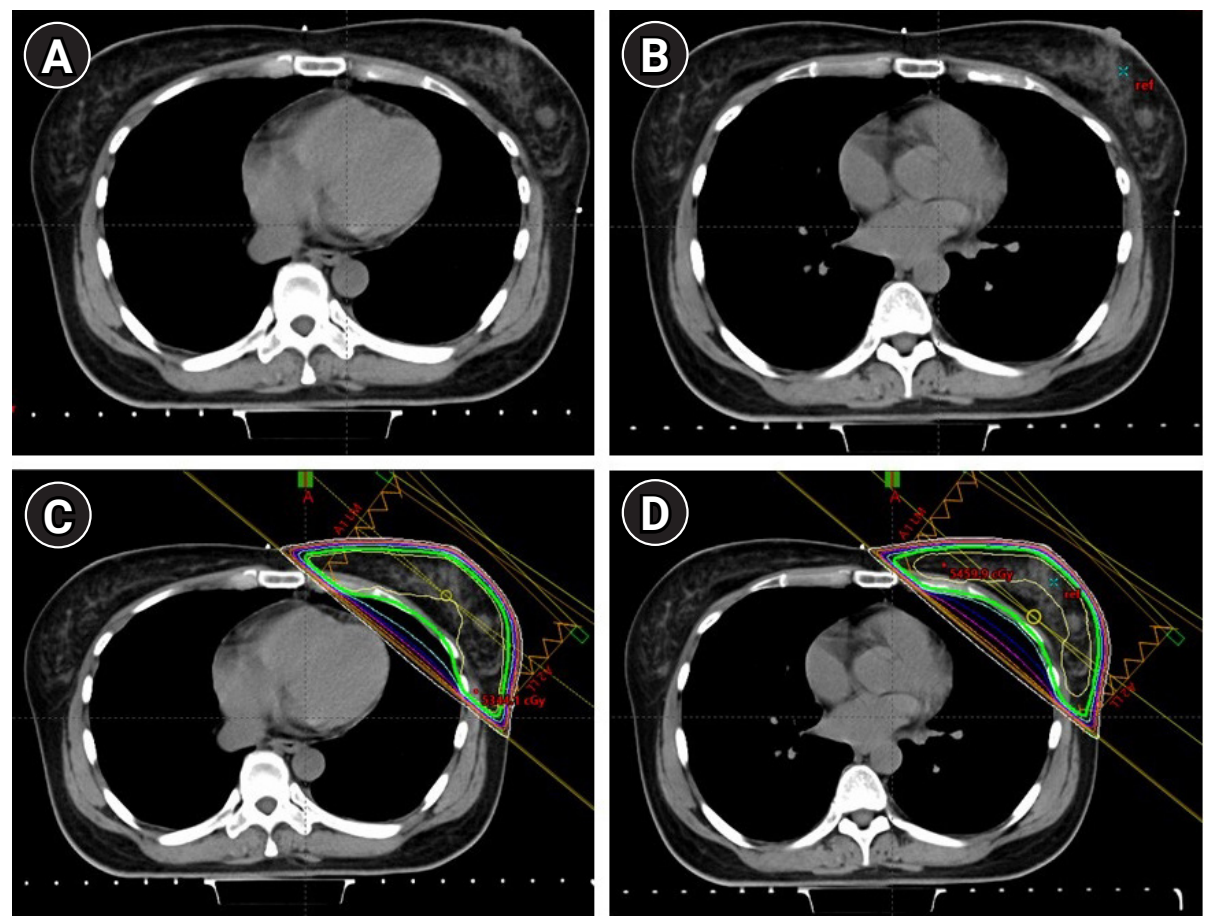

Fig. 1. Computed tomography (CT) simulation and treatment planning. CT simulation images of the FB technique (A) and the DIBH technique (B). Treatment fields and isodose distribution of the WB plan with the FB technique (C) and the DIBH technique (D). FB, free-breathing; DIBH, deep inspiration breath-hold; WB, whole breast. 
axial images were constructed with 3-mm spacing.

\section{Treatment planning}

Each patient underwent four treatment plans, covering the whole breast (WB) and WB plus IMNs of the first to third intercostal spaces (WB+IMNs) with both FB and DIBH techniques. To make the target volume coverages comparable, tangential whole breast radiotherapy (WBRT) fields with both breathing techniques utilized the same surface markers that defined the medial, lateral, superior and inferior borders (Fig. 1C, 1D). In WB+IMNs plans, clinical target volume (CTV) was delineated according to the Radiation Therapy Oncology Group (RTOG) breast contouring guidelines [15]. The planning target volume (PTV), 5-mm expansion from the CTV, was used to define the treatment field (Fig. 2). The WB+IMNs plans were normalized until 95\% of PTV receiving at least 45 Gy. Additionally, $95 \%$ of the target volume dose had to be no more than 1 Gy different between the FB and DIBH plans, as shown in dose volume histogram in Fig. 3. Forward intensity- modulated radiotherapy (IMRT) using an electronic compensator was applied in all plans. The prescription dose was $50 \mathrm{~Gy}$ in 25 fractions. The final dose distribution was calculated using Eclipse treatment planning system with inhomogeneity correction (Eclipse version 11.0.31). Normal structures including the heart, lungs and the LAD region were also contoured in both FB and DIBH images. The LAD region was contoured according to RADCOM atlas [16] (Fig. 4).

The cardiac parameters were mean LAD region dose, MHD, heart $V_{25}$, and heart $V_{40}$. The lung parameters included left lung $V_{20}$ and mean bilateral lungs. In-field maximum heart distance (maxHD), which was measured in centimeters on digitally reconstructed radiograph from CT images, was recorded and analyzed to determine whether this parameter correlated with MHD. The In-field maxHD values from all four plans, WB plans and WB+IMNs plans with both FB and DIBH techniques were used to study their correlations with the MHD. Breast volume (measured in $\mathrm{cm}^{3}$ ) from FB images of WB and WB+IMNs plan were used to study the correlation with the MHD.

Dosimetric parameters of the mean LAD region and heart and lung doses were compared using a two-tailed paired t-test. MaxHD and breast size were evaluated and analyzed using Spearman rank test to determine correlations with MHD.

\section{Results}

From March 2017 to November 2018, 25 left breast cancer patients who met the inclusion criteria were recruited. For both the WB and the WB+IMNs irradiation groups, compared with the FB
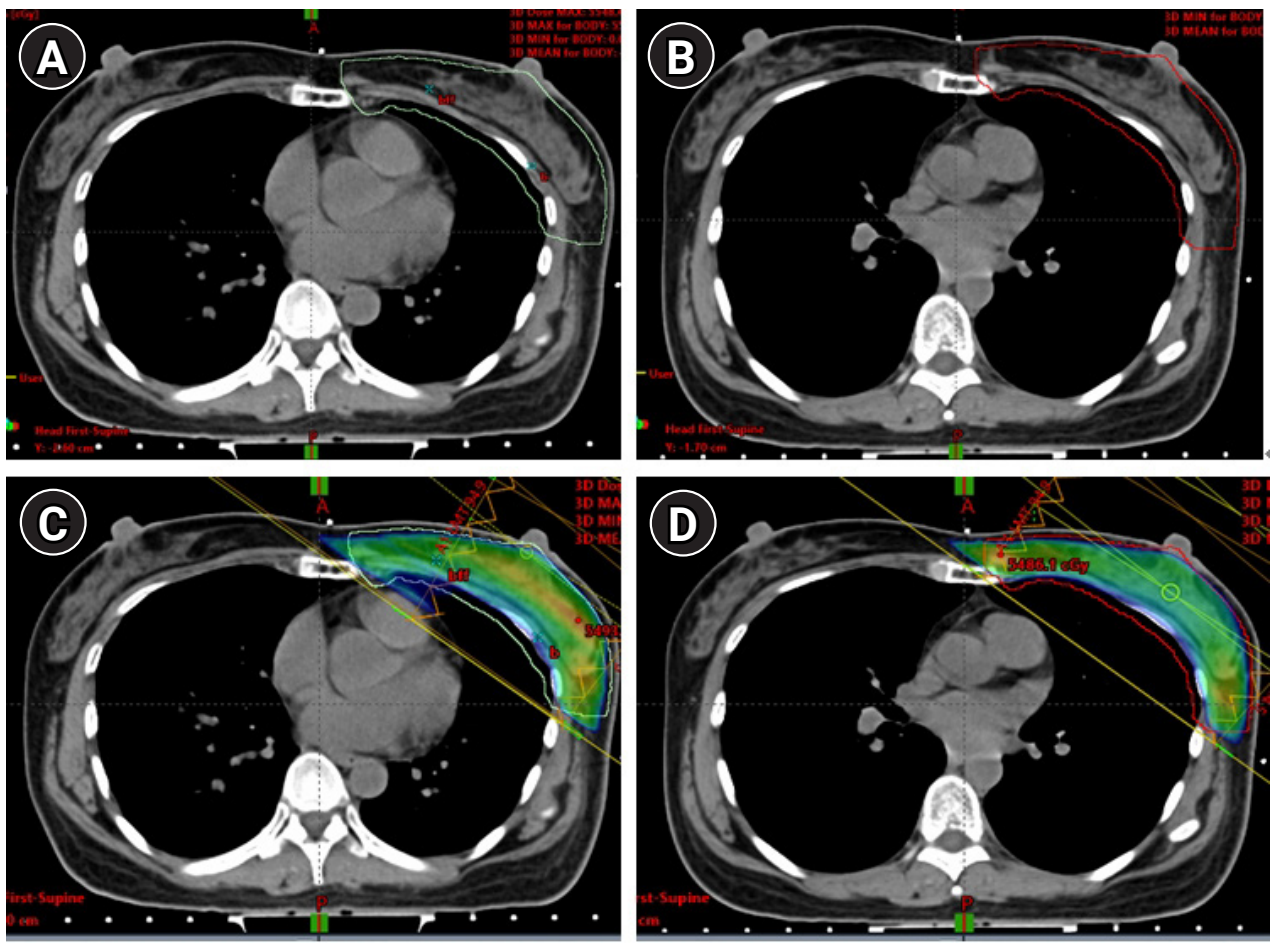

Fig. 2. Planning target volumes (PTVs) of the $W B+I M N s$ plan with the FB technique (A) and the DIBH technique (B). Treatment fields and the isodose distribution of the WB+IMNs plan with the FB technique (C) and the DIBH technique (D). WB, whole breast; IMN, internal mammary node; $F B$, free-breathing; DIBH, deep inspiration breath-hold. 

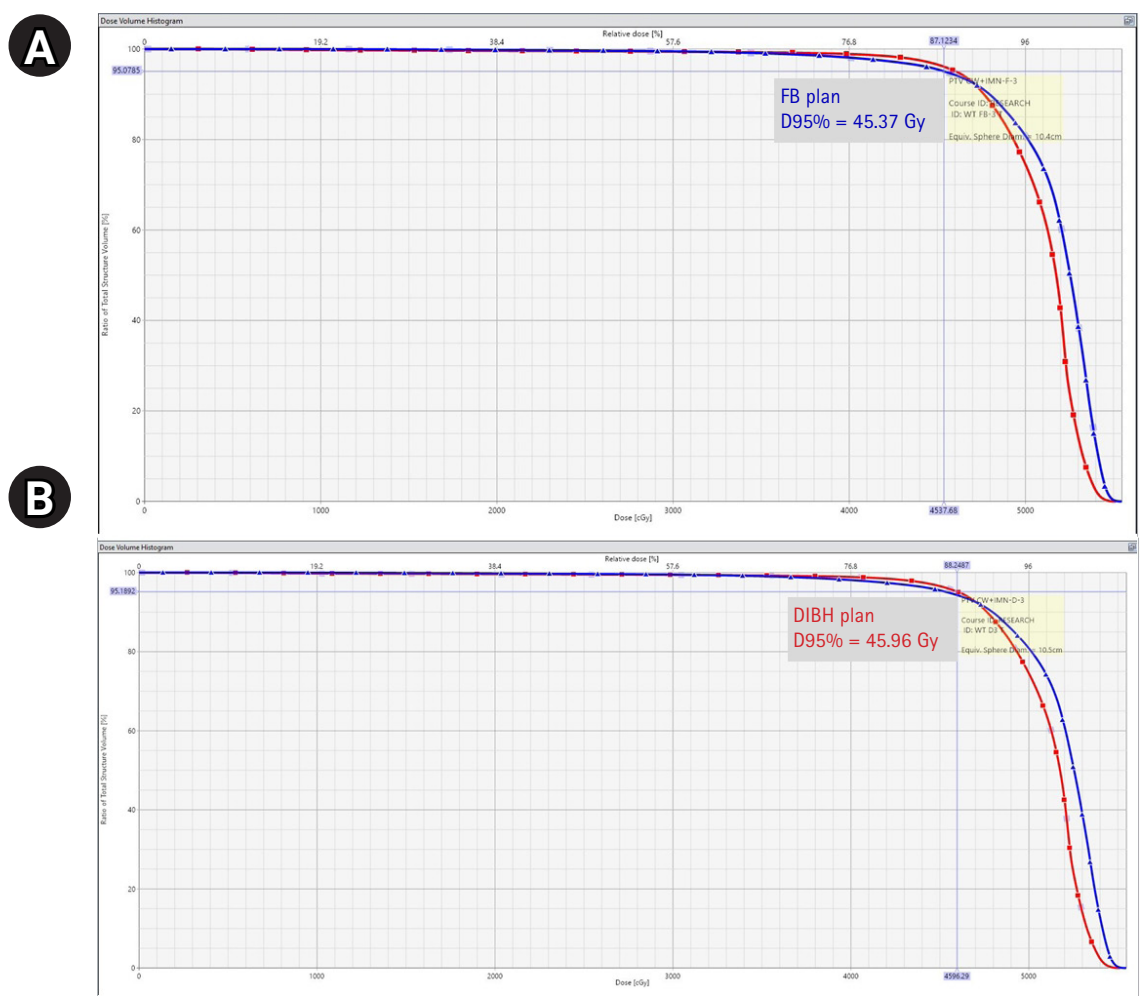

Fig. 3. Dose volume histogram of the planning target volume (PTV) of the WB+IMNs plan with the FB technique (A) and the DIBH technique (B). The WB+IMNs plans were normalized until 95\% of PTV received at least $45 \mathrm{~Gy}$. To be comparable, $95 \%$ of the target volume dose was no more than 1 Gy different between the FB and DIBH plans; this patient received 45.37 Gy and 45.96 Gy with the FB and DIBH plans, respectively. $W B$, whole breast; IMN, internal mammary node; FB, free-breathing; $D I B H$, deep inspiration breath-hold.
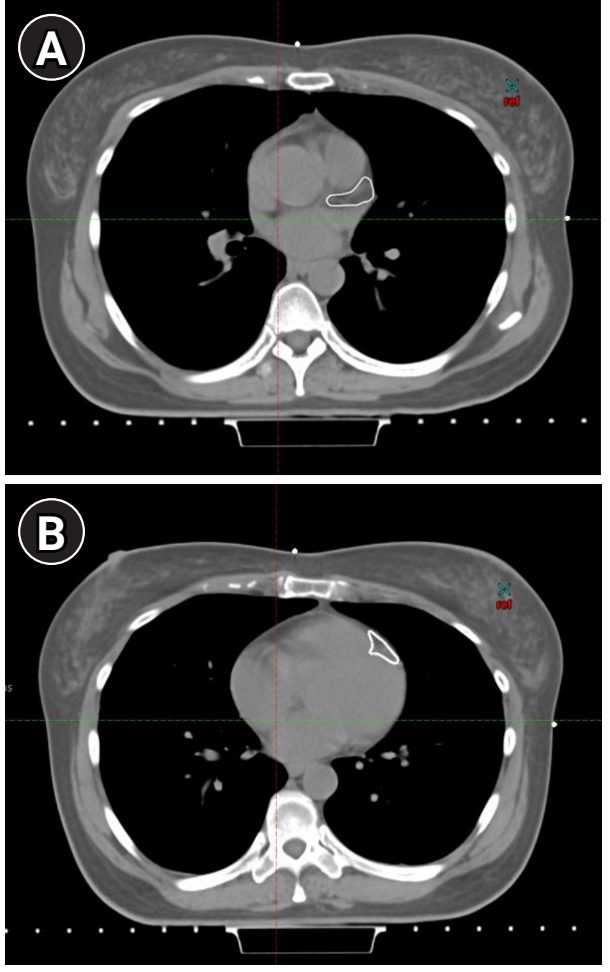

Fig. 4. The contouring method of the left anterior descending coronary artery (LAD) region. The anterior interventricular groove was contoured from the origin of the LAD (A) down to mid heart $(B)$ and continuing to the apex of the heart.
Table 1. Radiation doses to the normal tissues of the WB plans with the FB and DIBH techniques

\begin{tabular}{lccc}
\hline & \multicolumn{2}{c}{ Respiratory management } & \multirow{2}{*}{ p-value } \\
\cline { 2 - 3 } & DIBH & FB & \\
\hline Mean LAD region (Gy) & $11.48 \pm 8.1$ & $19.84 \pm 10.2$ & $<0.0001$ \\
& $(2.6-29.0)$ & $(4.0-45.0)$ & \\
Heart parameter & & & \\
Mean heart (Gy) & $2.95 \pm 2.3$ & $5.38 \pm 3.5$ & $<0.0001$ \\
& $(1.1-7.2)$ & $(2.0-14.0)$ & \\
Heart $\mathrm{V}_{25}(\%)$ & $3.48 \pm 4.4$ & $8.20 \pm 7.1$ & $<0.0001$ \\
& $(0.0-14.7)$ & $(1.4-25.0)$ & \\
Heart $\mathrm{V}_{40}(\%)$ & $2.48 \pm 3.7$ & $6.22 \pm 5.9$ & $<0.0001$ \\
& $(0.0-9.3)$ & $(0.0-21.0)$ & \\
Lung parameter & & & \\
Mean bilateral lungs $(\mathrm{Gy})$ & $5.02 \pm 1.0$ & $5.45 \pm 1.4$ & 0.07 \\
& $(3.6-7.0)$ & $(4.0-9.0)$ & \\
Left lung $\mathrm{V}_{20}(\%)$ & $19.72 \pm 4.3$ & $22.73 \pm 6.1$ & 0.0045 \\
& $(13.5-28.0)$ & $(14.5-39.0)$ & \\
\hline
\end{tabular}

Values are presented as mean \pm standard deviation (range).

WB, whole breast; FB, free-breathing; $\mathrm{DIBH}$, deep inspiration breathhold; LAD, left anterior descending coronary artery.

technique, the DIBH technique significantly reduced the radiation doses to all the heart parameters (Tables 1, 2). Reductions of the radiation doses to the lung parameters using the DIBH technique were observed in both plans. The dose reduction was only statistically sig- 
Table 2. Radiation doses to the normal tissues of the $\mathrm{WB}+\mathrm{IMNs}$ plans with the FB and DIBH techniques

\begin{tabular}{cccc}
\hline & \multicolumn{2}{c}{ Respiratory management } & \\
\cline { 2 - 3 } & DIBH & FB & \\
\hline Mean LAD region (Gy) & $23.88 \pm 10.6$ & $31.98 \pm 9.4$ & $<0.0001$ \\
& $(4.2-45)$ & $(9.8-50.0)$ & \\
Heart parameter & & & \\
Mean heart (Gy) & $6.43 \pm 3.8$ & $10.24 \pm 4.9$ & $<0.0001$ \\
& $(1.7-12.7)$ & $(3.9-19.9)$ & \\
Heart $\mathrm{V}_{25}(\%)$ & $10.37 \pm 8.0$ & $16.96 \pm 9.4$ & $<0.0001$ \\
& $(0.3-29.0)$ & $(4.6-38.0)$ & \\
Heart $\mathrm{V}_{40}(\%)$ & $7.50 \pm 6.0$ & $13.96 \pm 9.2$ & $<0.0001$ \\
& $(0.0-18.0)$ & $(2.4-34.0)$ & \\
Lung parameter & & & \\
Mean bilateral lungs (Gy) & $7.16 \pm 1.5$ & $7.45 \pm 1.6$ & 0.1 \\
& $(4.3-10.1)$ & $(4.7-10.5)$ & \\
Left lung $\mathrm{V}_{20}(\%)$ & $29.31 \pm 6.0$ & $32.10 \pm 6.3$ & 0.0009 \\
& $(20.0-42.0)$ & $(25.0-47.6)$ & \\
\hline
\end{tabular}

Values are presented as mean \pm standard deviation (range).

$W B$, whole breast; IMN, internal mammary node; $F B$, free-breathing; $\mathrm{DIBH}$, deep inspiration breath-hold; $L A D$, left anterior descending coronary artery.

nificant in the left lung $V_{20}$, and the mean bilateral lung doses were not significantly different between techniques (Tables 1, 2).

MHD correlated with the in-field maxHD $(r=0.925, p<0.001)$ as shown in Fig. 5A. Differences of MHD between the FB and DIBH techniques are also demonstrated in Fig. 5B. In addition, MHD also correlated with breast volume with correlation coefficients $(r)$ of $0.614, p=0.001$ and $r=0.642, p=0.0005$, in the WB and the WB+IMNs plans, respectively, as shown in Fig. 6. Notably, patients with larger breast sizes tended to receive a higher heart dose than those with smaller breast sizes.

\section{Discussion and Conclusion}

Advanced radiation technologies have contributed to the better treatment outcomes and longer survival of breast cancer patients. However, radiotherapy also has late treatment side effects, especially on the heart and coronary arteries in left-sided breast cancer patients. Therefore, recent studies have focused on techniques, especially the DIBH technique, that can reduce radiation doses to the heart and LAD.

In this study, we used an electronic compensator technique for the adjuvant radiation of breast cancer since this technique could achieve better homogeneity index as well as lower dose to the organs at risk [17]. Although the multileaf collimator could reduce cardiac dose, this study did not use it in order to assess the actual benefits of the DIBH technique over the FB technique. We found that the radiation doses to cardiac parameters were significantly reduced with the DIBH technique, similar to the results of previous

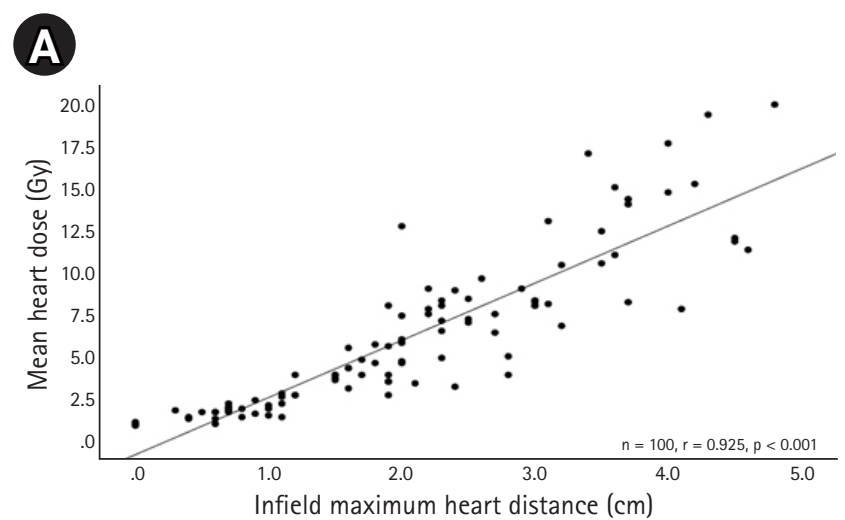

B

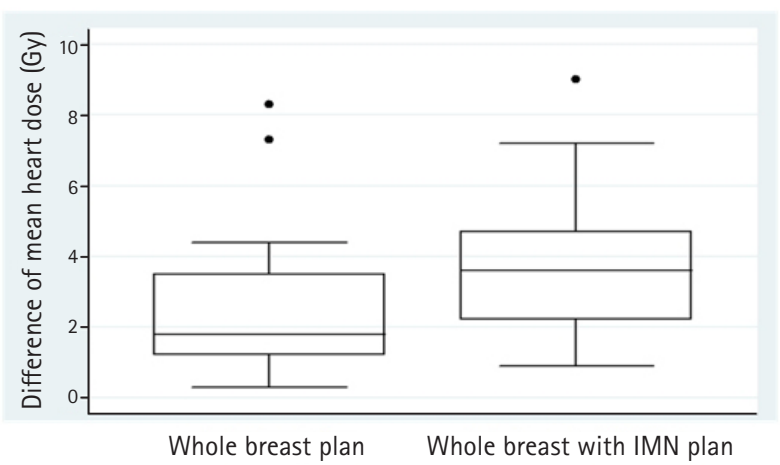

Fig. 5. (A) Correlation between in-field maximum heart distance and MHD in both the WB and WB+IMNs plans. (B) The differences of MHD between the FB and DIBH techniques (MHD of the FB - MHD of the DIBH) in both the WB and WB+IMNs plans. The horizontal lines above the boxes are the 75th percentile $+1.5 \times 10 R$. The upper border, the line inside, and the lower border of the box represent the 75th, 50th, and 25th percentiles, respectively. The horizontal lines below the boxes are the 25th percentile $-1.5 \times 10 R$. The three dots represent outliers ( $>75$ percentile $+1.5 \times I Q R)$. MHD, mean heart dose; $W B$, whole breast; IMN, internal mammary node; $F B$, free-breathing; $\mathrm{DIBH}$, deep inspiration breath-hold; IQR, interquartile range.

studies [9-11,18-21]. In the WB plans, the MHD was reduced from $5.38 \mathrm{~Gy}$ in FB to $2.95 \mathrm{~Gy}$ in DIBH (45.2\% reduction) (Table 1). Similarly, in the WB+IMNs plans, the MHD was also significantly reduced from 10.24 Gy with the FB technique to 6.43 Gy with the DIBH technique (37.2\% reduction) (Table 2 ).

In addition to MHD, volumes of the heart receiving high doses, heart $V_{25}$ and heart $V_{40}$, have been used to determine the radiation dose. In the Quantitative Analyses of Normal Tissue Effects in the Clinic (QUANTEC) study, a heart $\mathrm{V}_{25}$ of $<10 \%$ led to a less than $1 \%$ long-term cardiac mortality rate [22]. In our study, the heart $V_{25}$ was less than $10 \%$ with both the FB and the DIBH techniques for the WB plans. In the WB+IMNs plans, although the heart $\mathrm{V}_{25}$ with both the FB and DIBH techniques were more than 10\%, the value was reduced from $16.9 \%$ with the FB technique to $10.3 \%$ with the DIBH technique. 

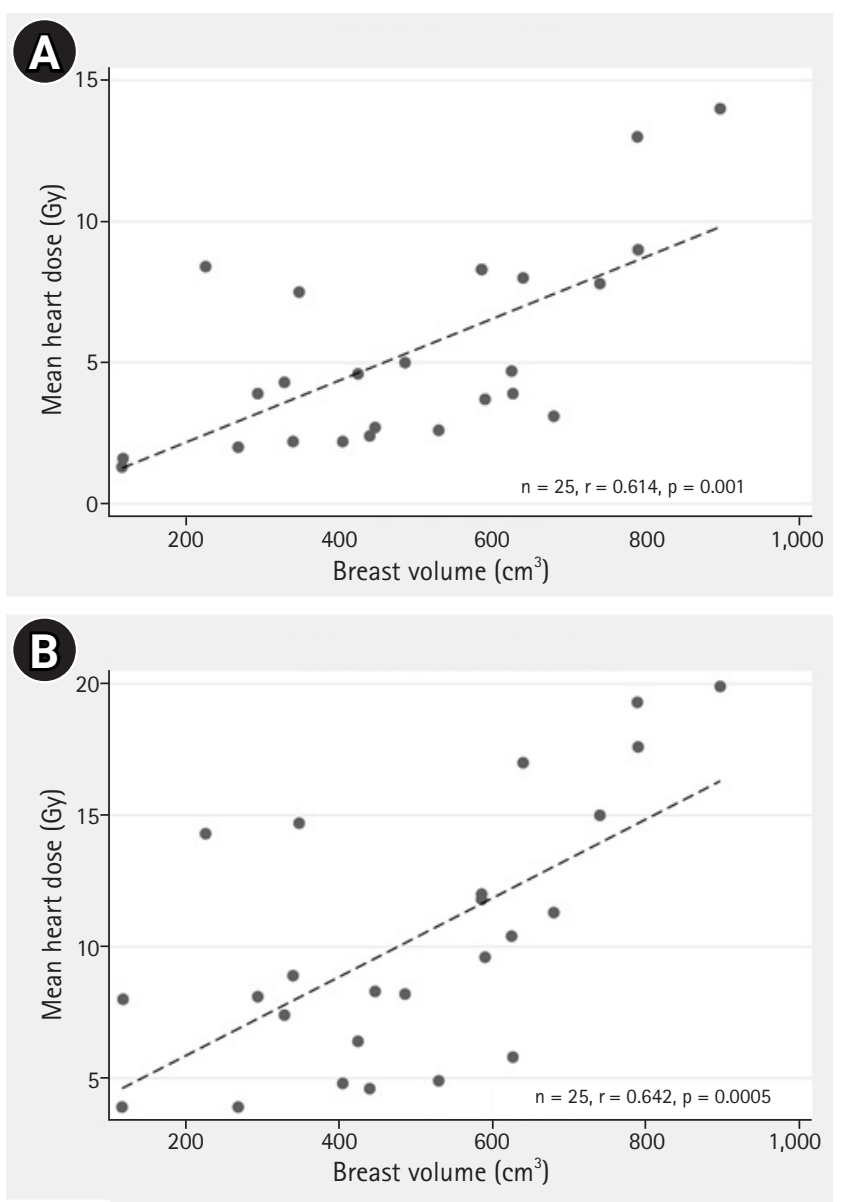

Fig. 6. Correlation between breast volume and mean heart dose with the $F B$ technique in the WB plan $(A)$ and the WB+IMNs plan (B). FB, free-breathing; WB, whole breast; IMN, internal mammary node.

Although the parameters of the radiation dose to the whole heart, including MHD, heart $\mathrm{V}_{25}$ and heart $\mathrm{V}_{40}$, have been widely used, radiation doses to other sub-structures of the heart, such as the $L A D$, have been proposed to be a potentially better parameter of coronary events. The BACCARAT study reported that the left ventricle and $L A D$ were the most radiation-exposed structures during left breast irradiation [8]. Therefore, the LAD dose may correlate with ischemic heart disease.

Previous studies found that the DIBH technique could reduce the radiation dose to the $\operatorname{LAD}[9,11,20]$. Contouring of the structures significantly affects the calculated radiation doses. Duane et al. [23] developed a cardiac contouring atlas for 15 cardiac segments, including 10 coronary arterial segments. However, the atlas needs contrast-enhanced images. After the contouring is standardized, the dose constraint to each structure can be developed. In our practice, we do not use radiation contrast; therefore, the LAD cannot be precisely determined. Instead we delineated the anterior interventricular groove from its origin down to the apex of the heart as the LAD region, according to the RADCOM study [16]. We found that in the WB plan, the radiation doses to the LAD region decreased from 19.84 Gy with the FB technique to 11.48 Gy with the DIBH technique (42.1\% reduction) (Table 1). Similarly, in the $W B+I M N s$ plans, the radiation doses also significantly reduced from 31.98 Gy with the FB technique to 23.88 Gy with the DIBH technique (25.3\%) (Table 2). Currently, the dose constraint to the $L A D$ region has not been determined. Some of our patients received mean LAD doses as high as 50 Gy (Table 2). Beaton et al. [24] reported that a maximum LAD dose of less than 45.4 Gy correlated with lower cardiac mortality. Since our study is a dosimetric study, a long-term follow-up study is needed to determine the clinical impact of the cardiac and LAD dose reductions.

Regarding lung doses, we found that all lung parameters were lower in the DIBH plans than in the FB plans (Tables 1, 2), similar to previous studies $[10,11,20]$. We did not include supraclavicular (SPC) field in the WB+IMNs plan in this study since the lung dose from SPC field would not have large differences between the FB and DIBH plans. The typical beam direction for the SPC field is anteroposterior, and the DIBH technique cannot reduce the irradiated lung volume. In addition, the volume and motion of the upper lobe are less affected by breathing than those of other lobes. However, in our clinical practice, we routinely irradiate the SPC field in all patients who receive radiation to IMNs.

In addition, we found that the maxHD correlated with MHD (Fig. $5 A)$, similar to a previous study [25]. Notably, to the best of our knowledge, our study is the first to show that breast size significantly correlated with MHD. Cardiac radiation exposure increased with larger breast sizes (Fig. 6). This is probably because large pendulous breasts would fall down posteriorly, forcing the posterior border of the tangential field to be wider.

In conclusion, the use of the DIBH technique in left breast cancer irradiation effectively reduces radiation exposure to the heart and $L A D$ region in both the WB and WB+IMNs plans. MHD correlates with the maximum heart distance and breast size.

\section{Conflict of Interest}

No potential conflict of interest relevant to this article was reported.

\section{References}

1. Imsamran W, Chaiwerawattana $A$, Wiangnon $S$, et al. Cancer in Thailand, 2010-2012. Bangkok, Thailand: New Thammada Press Co. Ltd.; 2015.

2. Ferlay J, Soerjomataram I, Dikshit R, et al. Cancer incidence and 
mortality worldwide: sources, methods and major patterns in GLOBOCAN 2012. Int J Cancer 2015;136:E359-86.

3. Early Breast Cancer Trialists' Collaborative Group (EBCTCG). Effect of radiotherapy after breast-conserving surgery on 10-year recurrence and 15-year breast cancer death: meta-analysis of individual patient data for 10,801 women in 17 randomised trials. Lancet $2011 ; 378: 1707-16$.

4. Early Breast Cancer Trialists' Collaborative Group (EBCTCG). Effect of radiotherapy after mastectomy and axillary surgery on 10year recurrence and 20-year breast cancer mortality: meta-analysis of individual patient data for 8135 women in 22 randomised trials. Lancet 2014;383:2127-35.

5. Thorsen LB, Offersen BV, Dano H, et al. DBCG-IMN: a population-based cohort study on the effect of internal mammary node irradiation in early node-positive breast cancer. J Clin Oncol 2016;34:314-20.

6. Chang JS, Park W, Kim YB, et al. Long-term survival outcomes following internal mammary node irradiation in stage II-III breast cancer: results of a large retrospective study with 12-year follow-up. Int J Radiat Oncol Biol Phys 2013;86:867-72.

7. Darby SC, McGale P, Taylor CW, Peto R. Long-term mortality from heart disease and lung cancer after radiotherapy for early breast cancer: prospective cohort study of about 300,000 women in US SEER cancer registries. Lancet Oncol 2005;6:557-65.

8. Jacob S, Camilleri J, Derreumaux S, et al. Is mean heart dose a relevant surrogate parameter of left ventricle and coronary arteries exposure during breast cancer radiotherapy: a dosimetric evaluation based on individually-determined radiation dose (BACCARAT study). Radiat Oncol 2019;14:29.

9. Smyth LM, Knight KA, Aarons YK, Wasiak J. The cardiac dose-sparing benefits of deep inspiration breath-hold in left breast irradiation: a systematic review. J Med Radiat Sci 2015;62:66-73.

10. Nissen HD, Appelt AL Improved heart, lung and target dose with deep inspiration breath hold in a large clinical series of breast cancer patients. Radiother Oncol 2013;106:28-32.

11. Vikstrom J, Hjelstuen MH, Mjaaland I, Dybvik KI. Cardiac and pulmonary dose reduction for tangentially irradiated breast cancer, utilizing deep inspiration breath-hold with audio-visual guidance, without compromising target coverage. Acta Oncol 2011;50:42-50.

12. Lai J, Hu S, Luo Y, et al. Meta-analysis of deep inspiration breath hold (DIBH) versus free breathing (FB) in postoperative radiotherapy for left-side breast cancer. Breast Cancer 2020;27:299-307.

13. Testolin A, Ciccarelli S, Vidano G, Avitabile R, Dusi F, Alongi F. Deep inspiration breath-hold intensity modulated radiation therapy in a large clinical series of 239 left-sided breast cancer pa- tients: a dosimetric analysis of organs at risk doses and clinical feasibility from a single center experience. Br J Radiol 2019; 92:20190150.

14. Chulalongkorn Hospital. Information for the patient: practical guide for deep inspiration breath hold (DIBH) in radiotherapy [Internet]. Bangkok, Thailand: Chulalongkorn Hospital; c2020 [cited 2020 Apr 17]. Available from: https://www.chulacancer. net/patient-list-page.php?id $=661$.

15. White J, Tai A, Arthur $D$, et al. Breast cancer atlas for radiation therapy planning: consensus definitions [Internet]. Philadelphia, PA: Radiation Therapy Oncology Group; c2020 [cited 2020 Aug 20]. Available from: https://www.rtog.org/CoreLab/ContouringAtlases/BreastCancerAtlas.aspx.

16. RADCOMP Consortium. Breast contouring RADCOMP Consortium 2016 [Internet]. Philadelphia, PA: Radiation Therapy Oncology Group; c2020 [cited 2020 Aug 20]. Available from: https://www. rtog.org/CoreLab/ContouringAtlases/RADCOMPBreastAtlas.aspx.

17. Nantavithya C, Saksornchai K, Oonsiri P, Shotelersuk K. Dosimetric study of three-dimensional conformal radiotherapy, electronic compensator technique, intensity-modulated radiation therapy and volumetric-modulated arc therapy in whole breast irradiation. J Radiother Pract 2017;16:431-43.

18. Stranzl H, Zurl B, Langsenlehner T, Kapp KS. Wide tangential fields including the internal mammary lymph nodes in patients with left-sided breast cancer. Influence of respiratory-controlled radiotherapy (4D-CT) on cardiac exposure. Strahlenther Onkol 2009;185:155-60.

19. Nguyen MH, Lavilla M, Kim JN, Fang LC. Cardiac sparing characteristics of internal mammary chain radiotherapy using deep inspiration breath hold for left-sided breast cancer. Radiat Oncol 2018;13:103.

20. Joo JH, Kim SS, Ahn SD, et al. Cardiac dose reduction during tangential breast irradiation using deep inspiration breath hold: a dose comparison study based on deformable image registration. Radiat Oncol 2015;10:264.

21. Hjelstuen MH, Mjaaland I, Vikstrom J, Dybvik KI. Radiation during deep inspiration allows loco-regional treatment of left breast and axillary-, supraclavicular- and internal mammary lymph nodes without compromising target coverage or dose restrictions to organs at risk. Acta Oncol 2012;51:333-44.

22. Gagliardi G, Constine LS, Moiseenko V, et al. Radiation dose-volume effects in the heart. Int J Radiat Oncol Biol Phys 2010;76(3 Suppl):S77-85.

23. Duane $F$, Aznar MC, Bartlett F, et al. A cardiac contouring atlas for radiotherapy. Radiother Oncol 2017;122:416-22.

24. Beaton $L$, Bergman $A$, Nichol $A$, et al. Cardiac death after breast radiotherapy and the QUANTEC cardiac guidelines. Clin Transl 
Radiat Oncol 2019;19:39-45.

25. Kong FM, Klein EE, Bradley JD, et al. The impact of central lung distance, maximal heart distance, and radiation technique on the volumetric dose of the lung and heart for intact breast radiation. Int J Radiat Oncol Biol Phys 2002;54:963-71. 